\title{
Pavane for a pulse pressure variation defunct
}

\author{
Soren Sondergaard
}

\begin{abstract}
Hemodynamic management of critically ill patients in the ICU or high-risk patients in the operating room has paradoxically shown progress in terms of outcome after the systematic application of volume responsiveness/ flow optimization based on pulse pressure variation and/or stroke volume variation during controlled, positive-pressure ventilation in patients without spontaneous respiratory efforts. This assessment of circulatory optimization should ideally be based on an exhaustive, predictive and coherent physiological understanding of the cardiovascular system model. This paper sketches the extremely complex physiological background of the concept of volume responsiveness, concluding that it is not a reliable means of guiding hemodynamic optimization because it is based on a nonexhaustive, nonpredictive and incoherent physiological model.
\end{abstract}

I wonder if the gentleman in the audience who denounces PPV still uses leeches in his practice? (Lecturer in reply to question concerning pulse pressure variation at ESICM Congress in Paris 2012)

\section{Introduction}

Pulse pressure variation (PPV) is promoted in the literature and at international meetings as a means of optimizing flow in patients subjected to correction of hemodynamic parameters in intensive care or in the operating room. Perel and colleagues [1] extended the work of Morgan and colleagues [2] and introduced the concept of systolic pressure variation, analyzing the relation of dUp and dDown in response to positive-pressure ventilation and intravascular volume expansion (VE). Focus shifted from systolic pressure to the amplitude of arterial pressure and the variation induced by positive-pressure ventilation [3]. Investigations into PPV have increased dramatically since 2000 (see Figure 1).

Correspondence: sondergaard.soren@gmail.com

Department of Anaesthesiology and Intensive Care Medicine, Sahlgrenska

University Hospital, University of Gothenburg, Blå Stråket 5,5, 41345,

Gothenburg, Sweden
The majority of studies have shown a distinct relationship between PPV and the prospect of increasing cardiac output (CO) by VE. Michard and Teboul suggested an explanation for this: PPV is seen as a reflection of the patient's heart function on the Starling curve, responding to VE (diminishing PPV) if on the ascending limb and nonresponding (no change in PPV) if on the level part of the cardiac function curve [4] (see Figure 2).

PPV is calculated according to the following equation:

$$
P P V, \quad \%=\frac{\left(P P_{\max }-P P_{\min }\right) \times 100}{\left(P P_{\max }+P P_{\min }\right) \times 0.5}
$$

where $\mathrm{PP}_{\max }$ is maximum pulse pressure and $\mathrm{PP}_{\min }$ is minimum pulse pressure. Usually a threshold value (derived from analysis of receiver operating characteristics) of PPV $>12$ to $16 \%$ is applied for the binary decision of whether the patient may be expected to be a responder or a nonresponder to VE in terms of increase in CO. PPV is a dynamic parameter in the sense that it demands an excitation of the cardiopulmonary system to deliver the signal of variation in pressure amplitude. The excitation is the cyclic variation in pleural pressure in positive-pressure ventilation. PPV has a number of related, derived dynamic measures: pulsoximetric plethysmographic variation, stroke volume variation, variation of the electrocardiography pre-ejection period, and a number of dynamic parameters derived from echocardiographic examination (for example, inferior caval vein diameter and collapsibility, left ventricular end diastolic area, respiratory variation of velocity time index of right and left ventricle or response of these measures to passive leg raising).

These measures may be regarded conceptually as system excitation-system response, the cardiopulmonary system representing a black box. In the following, PPV is used as an example for elucidation of some of the physiological mechanisms and to expose the contents of the black box. The reader is further referred to the excellent review by Magder including repository figures [5] and to Michard and colleagues' correspondence following Magder's review [6]. 


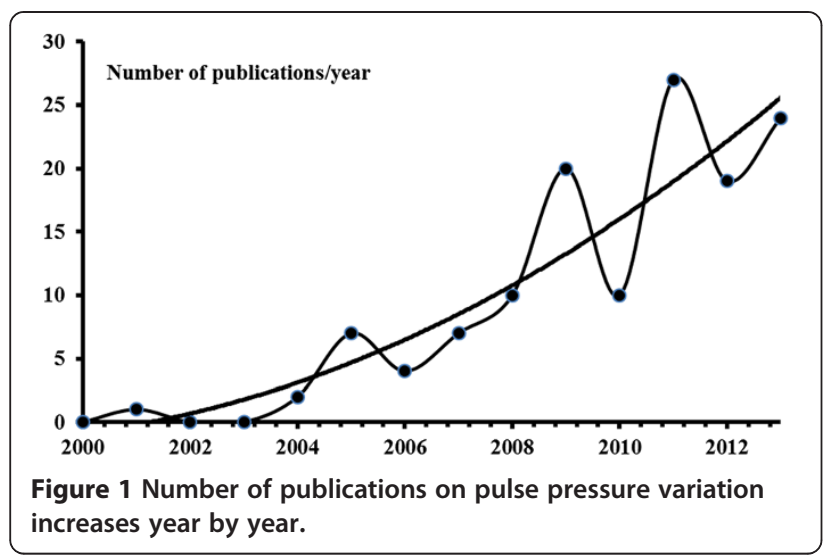

\section{Elucidation}

The working principle of PPV will be elucidated on the background of the combined venous return (VR) and cardiac function curves (see [7]). A cyclic change in pleural pressure is generated in positive-pressure ventilation, increasing during insufflation and waning during expiration. With each unit increase in pleural pressure, VR is inhibited by way of the transient increase in central venous pressure (CVP) or right atrial pressure (RAP). Depending on pulmonary compliance, the increase in pleural pressure increases RAP proportionally $1 \mathrm{mmHg}$ per $3 \mathrm{mmHg}$ insufflation pressure $[8,9]$. This decreases the difference from the mean systemic filling pressure $\left(\mathrm{P}_{\mathrm{ms}}-\mathrm{RAP}\right)$. This difference is one determinant of VR and, in turn, CO. $\mathrm{P}_{\mathrm{ms}}$ is affected by the cyclic change in pulmonary pressure

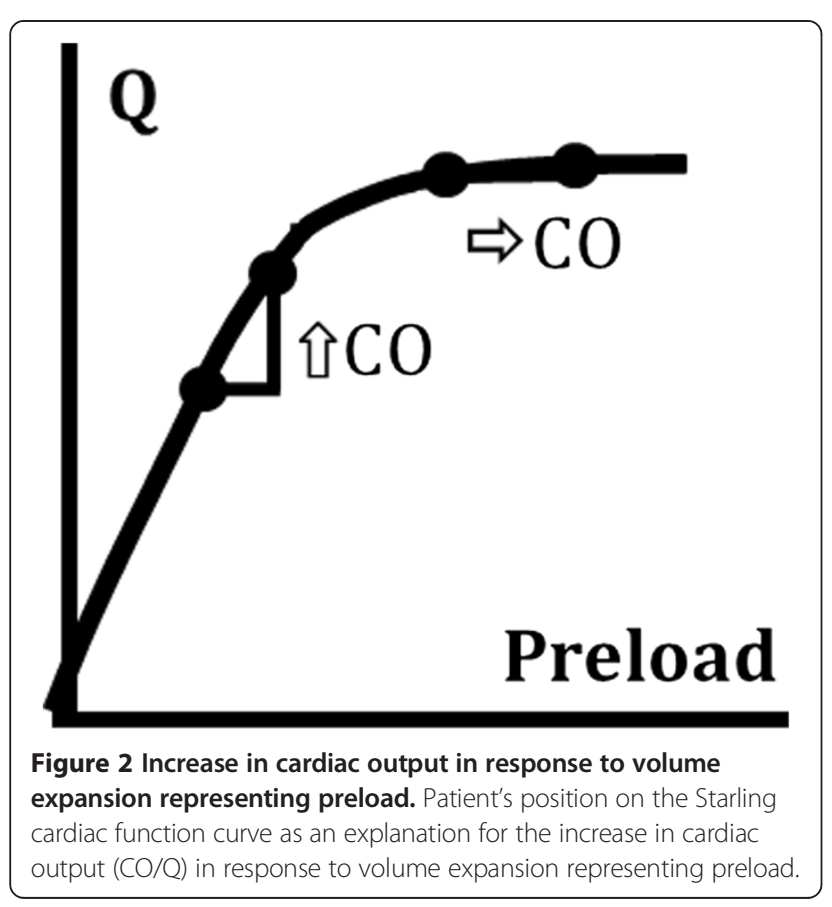

[10] and adapts to a constant increase as in positive end-expiratory pressure (PEEP) [11]. Decreasing difference $\left(\mathrm{P}_{\mathrm{ms}}-\mathrm{RAP}\right)$ diminishes the right ventricular stroke volume (RVSV) in the volume-responsive patient (in the words of the PPV concept), whereas this is not the case in the nonresponsive patient. This is illustrated in Figure 3.

\section{Circulatory factors}

One should contemplate the factors governing transmission of varying RVSV to variation in arterial pressure. As a first step, RAP is dissipated in two impedances, the resistance of the tricuspid valve and the elastance of the right ventricle. The right ventricular pressure, in turn, is dissipated into resistance of the pulmonary valve and the compliance and resistance of the pulmonary circulation - in short, the varying VR is manifested as pressures in four chambers depending on their elastances, as flow in four valves depending on their resistances and as pressure and flow in two vascular systems (pulmonary, systemic) depending on their compliances and resistances. If anything abnormal is occurring in any of these 10 relay stations, the signal will be distorted in unpredictable and undetectable ways.

Wyler von Ballmoos and colleagues demonstrated how pulmonary hypertension and right ventricular dysfunction in postcardiac and septic patients invalidated the determination of volume responsiveness [12]. The Bern group replicated this finding in an endotoxemic animal model, although still speculating about the relative contribution of pulmonary hypertension and right ventricular failure [13]. Michard and colleagues suggested that this lack of efficiency of PPV helped the clinician suspect right ventricular failure [14], and Mahjoub and colleagues recommended that the peak systolic velocity of tricuspid annular motion be assessed by tissue Doppler echocardiography in patients with PPV $>12 \%$ to detect false-positive PPV due to right ventricular dysfunction before fluid challenge [15]. Mesquida and colleagues likewise demonstrated in a canine study that PPV decreased when cardiac contractility was depressed by $\beta$-blockade, mimicking heart failure [16].

The pulmonary circulation acts as a capacitance vessel and the time-varying RVSV is subjected to at least three mechanisms during the passage from right to left heart through the pulmonary circulation. First, pleural pressure increases afterload on the right ventricle and preload on the left ventricle during insufflation. The impact of varying pleural pressure on the left atrium and left ventricle is minor compared with the effect on the right atrium and right ventricle because the pulmonary vasculature, left atrium and left ventricle are equally affected by the pleural pressure but the encroachment on right ventricular volume by the insufflation pressure increases left ventricular diastolic compliance. Second, during inspiration blood is squeezed from the pulmonary venous circulation to the left 

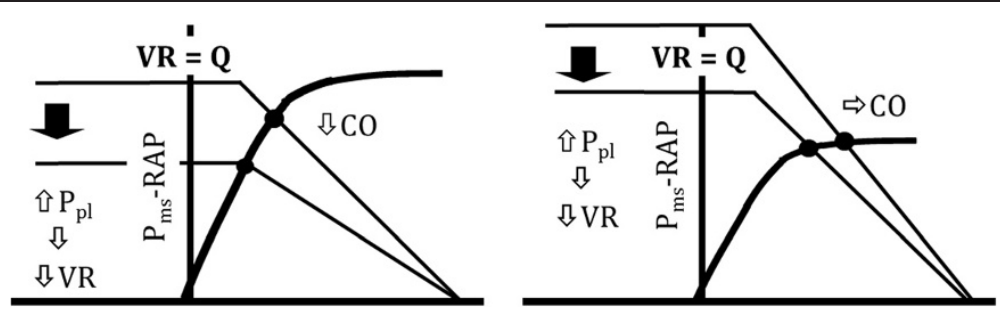

Figure 3 Difference between mean systemic filling and right atrial pressure correlates with venous return. With increasing pleural pressure $\left(\mathrm{P}_{\mathrm{pl}}\right)$ during inspiration, right atrial pressure (RAP) increases, diminishing the difference between mean systemic filling and right atrial pressure ( $\left.P_{m s}-R A P\right)$. CO/Q, increase in cardiac output; VR, venous return.

atrium. Finally, the passage of the RVSVs is determined by the cardiopulmonary transit time (CPTT), also known as the mean transit time. Michard and Teboul indicated that the changes in RVSV are transmitted 'après un délai de deux à trois battements cardiaques en raison du temps de transit sanguin pulmonaire prolongé' [17]. The CPTT, however, is anywhere from 4 to 14 seconds [18]. The CPTT/ mean transit time is dependent on the pulmonary blood volume, stroke volume and heart rate. These mechanisms have been summarized in rich detail by Robotham and colleagues in canine experiments with closed chest and intact heart and in a right heart bypass model [19]. With regard to the second item above, they noted that the increase in left ventricular stroke volume in certain conditions of PEEP and volume state was asynchronous with the increase in esophageal pressure (a surrogate for pleural pressure) and that other mechanisms must be at play. Robotham and colleagues included the respiratory rate, inspiratory/expiratory ratio, functional residual capacity, tidal volume, pulmonary blood volume and pulmonary vascular compliance as determinants for the arterial pressure amplitude. Consequently, the sequence of RVSVs per inspiratory/expiratory cycle may arrive in or out of phase with the respiratory rate and result in constructive or destructive interference. This phenomenon was illustrated by De Backer and colleagues [20] in accordance with the prediction of Robotham and colleagues.

A seemingly unexplored field is the establishment of threshold values for PPV dependent on age. As Magder notes: 'aortic elastance varies with age and disease and so will the relationship of stroke volume to pulse pressure. For a given $\mathrm{Q}$, stroke volume also varies with heart rate and therefore so will pulse pressure. Thus, the relationship of stroke volume to pulse pressure varies widely in the population, and this greatly limits quantitative predictions' [5]. Almost needless to say, the PPV concept only works for patients in sinus rhythm. Worth noting is that the occasional patient with ventricular premature complexes followed by compensatory pause opens the interesting opportunity to calculate the CPTT by analyzing arterial pressure amplitude (personal observation).

\section{Pulmonary factors}

Mechanics of the pulmonary system add to the complexity of transmission. de Waal and colleagues thus demonstrated, unsurprisingly, that the PPV concept does not work in open chest conditions because no pleural pressure changes are generated [21]. Undeniably, the patient must be without spontaneous respiratory effort as this interferes with the controlled, cyclic variation in intrathoracic pressure. If necessary, the patient must be heavily sedated or muscle relaxed, adding the onus of critical illness myopathy to critical disease.

The excitation must attain a pressure large enough to affect VR. This has been documented by several investigators, thus Muller and colleagues stated that the insufflation pressure must exceed $20 \mathrm{cmH}_{2} \mathrm{O}$ for the threshold value of $13 \%$ to be validly indicating volume responsiveness [22] and De Backer and colleagues argued that the tidal volume must exceed $8 \mathrm{ml} / \mathrm{kg}$ for PPV to reliably indicate volume responsiveness at a threshold value of $12 \%$ [23]. Mesquida and colleagues [16] replicated this finding by measuring PPV at increasing tidal volumes $(5,10,15$, and $25 \mathrm{ml} / \mathrm{kg}$ ) with resultant increasing PPV. The same group decreased thoracic wall compliance (and, as a corollary, total compliance) by restricting chest wall motion, again increasing PPV and changing the threshold value. Schmitt and colleagues demonstrated respiratory variations in the right ventricular stroke index estimated from the right ventricle outflow tract (respiratory variation of velocity time index of right ventricle) between 10 and $26 \%$ related to PEEP in human studies mirroring PEEPdependent RVSV as described in the above references [24]. Magder pointed out that lung compartments in West zone II $\left(\mathrm{P}_{\mathrm{a}}>\mathrm{P}_{\mathrm{A}}>\mathrm{P}_{\mathrm{v}}\right)$ during intermittent positive pressure ventilation (IPPV) decrease RVSV and cause significant PPV, but that this is unconnected to the volume state [25]. Renner and colleagues demonstrated that PPV was still valid in patients with increased abdominal pressure [26] but the cutoff value was $\geq 20.5 \%$ ! Freitas and colleagues described a threshold of $6.5 \%$ in PPV in septic patients ventilated in lung-protective mode with high PEEP [27]. Other thresholds are published in well circumscribed 
groups of patients (for a review see [28]). Smith and colleagues likewise described the effects of PEEP and called for a standardization of ventilatory settings when assessing volume responsiveness by PPV [29].

Recently, interest has been devoted to the phenomenon of respiratory sinus arrhythmia (see [30]). Respiratory sinus arrhythmia manifests in spontaneously breathing subjects as an increase in heart rate during inspiration and a decrease during expiration (positive respiratory sinus arrhythmia). This pattern is partly reversed, partly abolished in controlled IPPV (negative respiratory sinus arrhythmia). The impact of negative respiratory sinus arrhythmia dependent on ventilator settings and the hemodynamic state still has to be described.

\section{Experimental studies in pulse pressure variation}

The documentation for the use of PPV usually is headed as prediction of volume responsiveness based on a strict protocol involving a baseline measurement of $\mathrm{PPV} / \mathrm{CO}$ after 10 to 15 minutes of stable condition in terms of heart rate, $\mathrm{CO}$, mean arterial pressure and vasoactive and cardioactive medication followed by VE, usually 250 to $500 \mathrm{ml}$ colloid, delivered in 5 to 15 minutes followed by another calculation of $\mathrm{PPV} / \mathrm{CO}$, in total up to 30 minutes where nothing else can happen in terms of anesthetic management, surgical procedure or intensive care. Add to this the time course for the viscoelastic characteristics of the circulation to reach a new equilibrium [31]. This procedure demands some patience on the behalf of the surgeon to have his work interrupted for repeated 30-minute periods, and should anything happen during this interval affecting $\mathrm{RAP}$, mean arterial pressure or $\mathrm{CO}$, the measurement sequence is invalidated because this will affect the difference $\left(P_{m s}-R A P\right)$ and VR. Gelman enumerates at least eight variables affecting CVP: intra-abdominal pressure, cardiac pump function, pericardial pressure, pulmonary arterial pressure, venous resistance, intrathoracic pressure, stressed/unstressed volume and hypovolemia [32]. None of these can change during the 30-minute assessment of PPV! Three other characteristics of the PPV sequence are worth noticing. First, PPV can be eliminated by 5 to $10 \mathrm{mg}$ ephedrine and established by a modest infusion of nitroglycerine - changing the effective circulating volume, also known as stressed volume. This distinction between stressed and unstressed volume, however, is absent from the PPV concept. Cecconi and Rhodes, as a rare exception, drew attention to the fact that vascular tone may change during the volume challenge, invalidating the assessment of volume responsiveness [33]. Second, the fluid bolus must be standardized according to patient characteristics and vascular compliance (the suggestion of Smith and colleagues [29] to standardize ventilatory settings). Finally, and most importantly, PPV does not provide an assessment of optimum flow; it provides an estimate of whether flow can be increased by VE. The decision is binary defined by a threshold value derived from receiver operating characteristic analysis, although this has recently been slightly modified by the introduction of 'gray zones' (see Cannesson and colleagues [34] and Le Manach and colleagues [35]). Nothing is predicated of the patient's need for increased flow. There is general agreement on this (see Magder [5,25], Pinsky [36], Takala [37]). The threshold value is chosen to represent the - perceived - best combination of sensitivity and specificity, and considerations of cost/benefit of threshold are absent.

The PPV assessment is dependent on ventilatory settings, vasoactive and cardioactive medication. This may have induced the negative outcome of a study of goal-directed therapy: physically fit patients were volume resuscitated after induction of anesthesia - which rendered them volume responsive - but were not in need of volume. The surplus volume caused all of the well-known complications in the postoperative period (see Challand and colleagues [38]). A similar interpretation is possible in a study by Bundgaard-Nielsen and colleagues, who estimated the functional intravascular volume deficit in anesthetized patients by maximizing volume according to PPV $<10 \%$ [39]. The volume resuscitation was interpreted as the functional intravascular volume deficit. Physiologically intravascular volume was demobilized to an unstressed state by anesthetic vasodilation.

Pinsky described the incorporation of PPV into functional hemodynamic monitoring in circulatory unstable patients: 'If a patient responds to volume challenge or any equivalent preload responsiveness tests [PPV, plethysmographic variation in IPPV, variations in CVP in spontaneous ventilation, passive leg raising], then volume resuscitation should be initiated. In preload responsive patients who, despite increase in $\mathrm{CO}$ in response to fluid resuscitation, cannot maintain an adequate perfusion, a decrease in vascular tone should be suspected and vasopressors should be given in addition to fluids. If a hypotensive patient is neither preload responsive nor exhibiting reduced vasomotor tone, then the problem is the heart or an obstruction in blood flow such as thromboembolic events' [36]. This is the sequential approach to resuscitation. The suggested volume challenge carries a success rate of approximately $50 \%$ in terms of increase in $\mathrm{CO}$ and an undeclared risk of volume overloading the patient, as summarized by Marik and colleagues: 'Whereas under-resuscitation results in inadequate organ perfusion, accumulating data suggest that overresuscitation increases the morbidity and mortality of critically ill patients' [40]. Still, nine years later, nobody has answered Professor Pinsky's call: 'No study yet published has prospectively predicted which patients would be preload responsive based on these measures and then shown that their predictions were correct' [41]. The choice of therapy, alternatively, may be supported by performing 
one of the equivalent preload responsiveness tests, which would obviate the need for $\mathrm{CO}$ measurement. So on the one hand there is a volume challenge with a $50 \%$ chance of improving $\mathrm{CO}$ and an unknown risk of overloading the patient, and on the other there is a preload responsiveness test based on complex cardiopulmonary interaction - and neither approach answers the question of why the patient is circulatory unstable or whether the patient is in need of volume for optimization. MacLean and Duff in [42] argued the case for CVP in volume resuscitation in shock, adducing three causes for circulatory instability: cardiac failure, volume deficit and peripheral vascular failure. We may think differently about the utility of CVP, but the three causes stand the test of time (see Payen [43]). Alternatively, the simultaneous measurement of $\mathrm{CO}$, mean arterial pressure and CVP readily allows for clinical assessment of whether the cause is cardiac function, volume state and/or vasomotor tone. These factors are the determinants of cardiovascular function and, in a more comprehensive cardiovascular model, are obvious candidates for studying the simultaneous and judicious use of volume, vasoactive and cardioactive drugs in contrast to the sequential application in functional hemodynamic monitoring. This seems to be a reasonable venue of research in managing circulatory instable patients in surgery and intensive care.

In summary, PPV (and other reflections of cardiopulmonary interaction) is heralded as an assessment of optimizing the volume and flow state of patients in anesthesia and intensive care by analyzing variations in pressure amplitude as elicited by IPPV. From carefully controlled studies a threshold value is derived for the optimal sensitivity and specificity, and the clinician arrives at a binary decision of whether to expand volume. Firstly, the concept does not provide an optimum of volume/flow and all agree that a patient may be volume responsive but not in need of volume. Next, the concept is weighed down by the most intricate physiology of cardiopulmonary interaction, which makes it impossible to ascertain whether a positive or a negative result of the PPV calculation actually indicates volume responsiveness. Third, the literature abounds in threshold values defined in various patient populations under various conditions spanning from 6 to $20 \%$. Finally, the subject is an exceptional demonstration of physiology but hardly guides the management of critically ill patients.

\section{Proposed checklist before performing volume expansion suggested by PPV}

$\checkmark$ Is the patient ventilated with IPPV without spontaneous efforts?

$\checkmark$ Is the patient ventilated in nonprotective ventilation (tidal volume at least $8 \mathrm{ml} / \mathrm{kg}$ )?

$\checkmark$ Is the patient in sinus rhythm? $\checkmark$ Is chest wall compliance normal (thorax closed, no flail chest, no binding)?

$\checkmark$ Is the patient unaffected by valvular disease?

$\checkmark$ Is the patient unaffected by right ventricle and/or left ventricle dysfunction (for example, as assessed by an echocardiographic examination including measurement of peak systolic velocity of tricuspid annular motion assessed by tissue Doppler echocardiography)?

$\checkmark$ Does the patient have normal abdominal pressure?

$\checkmark$ Have you decided or have you performed a calibration to decide which threshold value ( 6 to $20.5 \%$ ) should be used for the binary decision of volume responsiveness?

$\checkmark$ Have you established the compliance of the patient's vascular capacitance in order to standardize the VE?

$\checkmark$ Have you established that the patient's heart rate/ respiratory rate ratio is $\geq 3.6$ ?

$\checkmark$ Can you safely establish a baseline, perform a volume challenge and remeasure without any factors affecting heart efficiency and/or vasomotor tone during the assessment?

\section{Abbreviations}

CO: Cardiac output; CPTT: Cardiopulmonary transit time; CVP: Central venous pressure; IPPV: Intermittent positive pressure ventilation; PEEP: Positive endexpiratory pressure; $P_{m s}$ : Mean systemic filling pressure; PPV: Pulse pressure variation; RAP: Right atrial pressure; RVSV: Right ventricular stroke volume; VE: Volume expansion; VR: Venous return.

\section{Competing interests}

The author declares that he has no competing interests.

\section{Published: 14 Nov 2013}

\section{References}

1. Perel A, Pizov R, Cotev S: Systolic blood pressure variation is a sensitive indicator of hypovolemia in ventilated dogs subjected to graded hemorrhage. Anesthesiology 1987, 67:498-502

2. Morgan BC, Martin WE, Hornbein TF, Crawford EW, Guntheroth WG: Hemodynamic effects of intermittent positive pressure respiration. Anesthesiology 1966, 27:584-590.

3. Michard F, Boussat S, Chemla D, Anguel N, Mercat A, Lecarpentier Y, Richard C, Pinsky MR, Teboul JL: Relation between respiratory changes in arterial pulse pressure and fluid responsiveness in septic patients with acute circulatory failure. Am J Respir Crit Care Med 2000, 162:134-138.

4. Michard F, Teboul JL: Using heart-lung interactions to assess fluid responsiveness during mechanical ventilation. Crit Care 2000, 4:282-289.

5. Magder S: Clinical usefulness of respiratory variations in arterial pressure. Am J Respir Crit Care Med 2004, 169:151-155.

6. Michard F, Chemla D, Teboul JL: More respect for respiratory variation in arterial pressure. Am J Respir Crit Care Med 2004, 169:1333-1334. author reply 1334.

7. Guyton AC: Determination of cardiac output by equating venous return curves with cardiac response curves. Physiol Rev 1955, 35:123-129.

8. Magder S: Central venous pressure monitoring. Curr Opin Crit Care 2006, 12:219-227.

9. Yang ZL, Zhou JQ, Sun BL, Qian ZX, Zhao H, Liu WD: The influence of positive end-expiratory pressure on central venous pressure in patients with severe craniocerebral injury. Zhongguo Wei Zhong Bing Ji Jiu Yi Xue 2012, 24:283-285.

10. Chihara E, Hashimoto S, Kinoshita T, Hirose M, Tanaka Y, Morimoto T: Elevated mean systemic filling pressure due to intermittent positivepressure ventilation. Am J Physiol 1992, 262:H1116-H1121.

11. Luecke T, Pelosi P: Clinical review: Positive end-expiratory pressure and cardiac output. Crit Care 2005, 9:607-621. 
12. Wyler von Ballmoos M, Takala J, Roeck M, Porta F, Tueller D, Ganter CC Schroder R, Bracht H, Baenziger B, Jakob SM: Pulse-pressure variation and hemodynamic response in patients with elevated pulmonary artery pressure: a clinical study. Crit Care 2010, 14:R111.

13. Daudel F, Tuller D, Krahenbuhl S, Jakob SM, Takala J: Pulse pressure variation and volume responsiveness during acutely increased pulmonary artery pressure: an experimental study. Crit Care 2010, 14:R122.

14. Michard F, Richards G, Biais M, Lopes M, Auler JO: Using pulse pressure variation or stroke volume variation to diagnose right ventricular failure? Crit Care 2010, 14:451. author reply 451.

15. Mahjoub Y, Pila C, Friggeri A, Zogheib E, Lobjoie E, Tinturier F, Galy C, Slama $M$, Dupont $H$ : Assessing fluid responsiveness in critically ill patients: false-positive pulse pressure variation is detected by Doppler echocardiographic evaluation of the right ventricle. Crit Care Med 2009, 37:2570-2575.

16. Mesquida J, Kim HK, Pinsky MR: Effect of tidal volume, intrathoracic pressure, and cardiac contractility on variations in pulse pressure, stroke volume, and intrathoracic blood volume. Intensive Care Med 2011, 37:1672-1679.

17. Michard F, Teboul JL: Évaluation des besoins volémiques en réanimation. Reanimation 2001, 10:162-172.

18. Lewis ML, Gnoj J, Fisher VJ, Christianson LC: Determinants of pulmonary blood volume. J Clin Invest 1970, 49:170-182.

19. Robotham JL, Cherry D, Mitzner W, Rabson JL, Lixfeld W, Bromberger-Barnea B: A re-evaluation of the hemodynamic consequences of intermittent positive pressure ventilation. Crit Care Med 1983, 11:783-793.

20. De Backer D, Taccone FS, Holsten R, Ibrahimi F, Vincent JL: Influence of respiratory rate on stroke volume variation in mechanically ventilated patients. Anesthesiology 2009, 110:1092-1097.

21. de Waal EE, Rex S, Kruitwagen CL, Kalkman CJ, Buhre WF: Dynamic preload indicators fail to predict fluid responsiveness in open-chest conditions. Crit Care Med 2009, 37:510-515

22. Muller L, Louart G, Bousquet PJ, Candela D, Zoric L, de La Coussaye JE, Jaber $S$, Lefrant JY: The influence of the airway driving pressure on pulsed pressure variation as a predictor of fluid responsiveness. Intensive Care Med 2010, 36:496-503.

23. De Backer D, Heenen S, Piagnerelli M, Koch M, Vincent JL: Pulse pressure variations to predict fluid responsiveness: influence of tidal volume. Intensive Care Med 2005, 31:517-523.

24. Schmitt JM, Vieillard-Baron A, Augarde R, Prin S, Page B, Jardin F: Positive end-expiratory pressure titration in acute respiratory distress syndrome patients: impact on right ventricular outflow impedance evaluated by pulmonary artery Doppler flow velocity measurements. Crit Care Med 2001, 29:1154-1158.

25. Magder S: Further cautions for the use of ventilatory-induced changes in arterial pressures to predict volume responsiveness. Crit Care 2010, 14:197.

26. Renner J, Gruenewald M, Quaden R, Hanss R, Meybohm P, Steinfath M, Scholz J, Bein B: Influence of increased intra-abdominal pressure on fluid responsiveness predicted by pulse pressure variation and stroke volume variation in a porcine model. Crit Care Med 2009, 37:650-658.

27. Freitas FG, Bafi AT, Nascente AP, Assuncao M, Mazza B, Azevedo LC, Machado FR: Predictive value of pulse pressure variation for fluid responsiveness in septic patients using lung-protective ventilation strategies. Br J Anaesth 2013, 110:402-408.

28. Cavallaro F, Sandroni C, Antonelli M: Functional hemodynamic monitoring and dynamic indices of fluid responsiveness. Minerva Anestesio/ 2008, 74:123-135.

29. Smith F, Geyser M, Schreuder I, Becker P: The effect of positive end-expiratory pressure on pulse pressure variation. South Afr J Anaesth Analg 2012. 18:333-338.

30. Beda A, Carvalho NC, Guldner A, Koch T, de Abreu MG: Mechanical ventilation during anaesthesia: challenges and opportunities for investigating the respiration-related cardiovascular oscillations. Biomed Tech (Berl) 2011, 56:195-206

31. Guyton AC, Lindsey AW, Kaufmann BN: Effect of mean circulatory filling pressure and other peripheral circulatory factors on cardiac output. Am J Physiol 1955, 180:463-468.

32. Gelman S: Venous function and central venous pressure: a physiologic story. Anesthesiology 2008, 108:735-748.

33. Cecconi $M$, Rhodes $A$ : Pulse pressure analysis: to make a long story short. Crit Care 2010, 14:175.
34. Cannesson M, Le Manach Y, Hofer CK, Goarin JP, Lehot JJ, Vallet B, Tavernier B: Assessing the diagnostic accuracy of pulse pressure variations for the prediction of fluid responsiveness: a 'gray zone' approach. Anesthesiology 2011, 115:231-241.

35. Le Manach Y, Hofer CK, Lehot JJ, Vallet B, Goarin JP, Tavernier B, Cannesson M: Can changes in arterial pressure be used to detect changes in cardiac output during volume expansion in the perioperative period? Anesthesiology 2012, 117:1165-1174.

36. Pinsky MR: Goal-Directed Therapy: Optimizing Fluid Management in Your Patient. http://www.initiatives-patientsafety.org/.

37. Takala J: Hemodynamic management of the critically ill patient: craft meets science. Curr Opin Crit Care 2007, 13:519-520.

38. Challand C, Struthers R, Sneyd JR, Erasmus PD, Mellor N, Hosie KB, Minto G: Randomized controlled trial of intraoperative goal-directed fluid therapy in aerobically fit and unfit patients having major colorectal surgery. Br J Anaesth 2012, 108:53-62.

39. Bundgaard-Nielsen $M$, Jorgensen $\mathrm{CC}$, Secher NH, Kehlet H: Functional intravascular volume deficit in patients before surgery. Acta Anaesthesiol Scand 2010, 54:464-469.

40. Marik PE, Monnet $X$, Teboul $J$ L: Hemodynamic parameters to guide fluid therapy. Ann Intensive Care 2011, 1:1.

41. Pinsky MR: Using ventilation-induced aortic pressure and flow variation to diagnose preload responsiveness. Intensive Care Med 2004, 30:1008-1010.

42. MacLean LD, Duff JH: The use of central venous pressure as a guide to volume replacement in shock. Chest 1965, 48:199-205.

43. Payen D: Prediction of fluid challenge effect: filling pressure when left ventricular function is abnormal, diastolic volume when left ventricular function is normal. Crit Care 2011, 15:139.

$10.1186 / \mathrm{cc} 13109$

Cite this article as: Sondergaard: Pavane for a pulse pressure variation defunct. Critical Care 2013, 17:327 